\title{
A Cable-Passive Damper System for Sway and Skew Motion Control of a Crane Spreader
}

\author{
La Duc Viet ${ }^{1}$ and Youngjin Park ${ }^{2}$ \\ ${ }^{1}$ Institute of Mechanics, Vietnam Academy of Science and Technology, 264 Doi Can, Hanoi 10000, Vietnam \\ ${ }^{2}$ Korea Advanced Institute of Science and Technology, Science Town, Daejeon 305-701, Republic of Korea \\ Correspondence should be addressed to La Duc Viet; ldviet@imech.ac.vn
}

Received 15 December 2014; Revised 2 April 2015; Accepted 3 April 2015

Academic Editor: Ahmet S. Yigit

Copyright (C) 2015 L. D. Viet and Y. Park. This is an open access article distributed under the Creative Commons Attribution License, which permits unrestricted use, distribution, and reproduction in any medium, provided the original work is properly cited.

While the crane control problem is often approached by applying a certain active control command to some parts of the crane, this paper proposes a cable-passive damper system to reduce the vibration of a four-cable suspended crane spreader. The residual sway and skew motions of a crane spreader always produce the angle deflections between the crane cables and the crane spreader. The idea in this paper is to convert those deflections into energy dissipated by the viscous dampers, which connect the cables and the spreader. The proposed damper system is effective in reducing spreader sway and skew motions. Moreover, the optimal damping coefficient can be found analytically by minimizing the time integral of system energy. The numerical simulations show that the proposed passive system can assist the input shaping control of the trolley motion in reducing both sway and skew responses.

\section{Introduction}

Because the crane spreader suspended by cables is highly flexible in nature, the external disturbances, such as wind or motion of the support unit (e.g., the bridge or trolley or tower) often cause the residual oscillation. In general, the control strategies proposed in the literature are often approached by applying control command to some parts of crane. The control command can be applied to the cables $[1,2]$, to the trolley $[3,4]$, to the boom $[5,6]$, or to the active mass damper [7]. The control algorithm can be based on open-loop or closedloop techniques [8]. The closed-loop (feedback) techniques use the crane measurements (e.g., deflection, and position) to generate the control command. The feedback control provides disturbance rejection but it is not easy to obtain accurate measurements of payload deflection and other system states. The sensors can be expensive and difficult to mount, calibrate, and maintain. Feedback control can also cause unexpected motions that make it difficult for the human operator to drive the crane. On the other hand, a typical open-loop technique, namely, input shaping, modifies the desired velocity command before it is issued to the crane motors [9-12]. The input shaping techniques are easy to apply and do not require the sensors. However, they lack the ability to handle external disturbances or initial conditions. While many other studies on open- and closed-loop control of crane can be referred to in the references of the aforementioned papers, this paper proposes a rather more conventional damping system.

It is well-known that the passive viscous damper is the most popular and simple device used in vibration control. Because the crane payload is often modeled as a single pendulum, it is not easy to imagine how to install a passive damper into a single cable. Some recent approaches propose the Coriolis damper to the rigid pendulum [13-15]. In fact, many actual crane mechanisms consist typically of a set of four hoisting cables dropping from four points on a trolley and attached to four points on a spreader bar. Some studies have considered multicables mechanism $[1,2,16,17]$. The idea of this paper is based on a reality that when the spreader oscillates, the angles between the cables and the spreader change. It is noted that if the spreader is treated as a point mass, the mentioned angles are meaningless. If we consider the actual multicables mechanism of the crane, the opportunity is opened for converting the relative movements between 


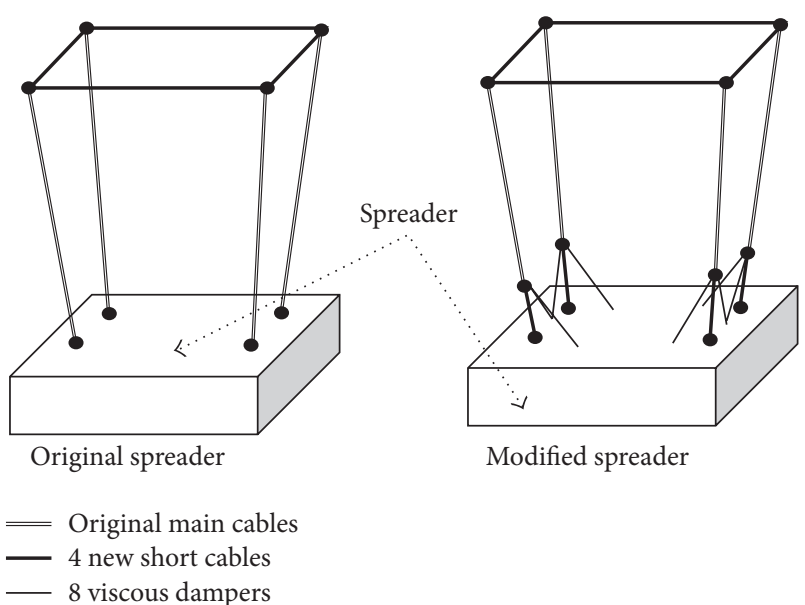

FIGURE 1: Modified spreader to implement the cable damper system.

the cables and the spreader into the energy dissipation in the viscous dampers. The proposed damping system in this paper is purely passive and it can not replace the active control schemes. Instead of that, because the passive devices do not rely on sensors and external energy, it is expected that the proposed damping system can be used to improve any active control scheme acting on the crane. To demonstrate this fact, in the numerical simulation in this paper, the proposed system is used to improve an input shaping control of the trolley motion.

In the multicables mechanism, the crane spreader has six degrees of freedom: three translational motions and three rotational motions. Three rotational motions consisting of roll, pitch, and yaw (skew) can be caused by the uneven distribution of the materials inside the container or misaligned ropes or other external disturbances like the wind or interaction of the load with other rigid obstacles. Sometimes they make it difficult to position the spreader fast and accurately. Among these three rotational motions, the skew motion is the most critical in the operation of a crane.

The novelty of this paper is the proposal and design of a cable-damper system which can regulate effectively both sway and skew motions of a four-cable suspended crane spreader. The cable damper system consists of 4 short cables and 8 viscous dampers connecting the crane long cables and the crane spreader through spherical joints (Figure 1). The structure of paper is as follows. At first, the full linearized equations of motion containing 18 equations are written in nondimensional vector matrix form. Then the formula of the stored energy of sway motion and skew motion are derived based on Lyapunov matrix equation. The optimal damping coefficient is obtained analytically by minimizing the stored energy. At last, the damper effectiveness is verified by nonlinear numerical simulations.

\section{Equations of Free Vibration}

The proposed cable-damper system consists of 4 new cables and 8 viscous dampers as shown in Figure 1. The original cables are proposed not to connect directly to the spreader but through the cable-damper system (Figure 1).

Let us consider the details in Figure 2.

Two coordinate reference frames are assigned. They are the global reference frame (Oxyz) (called T-frame), on the base of the rail, and the spreader attached reference frame $\left(P x_{s} y_{s} z_{s}\right)$ (called S-frame), in the geometric center of the top of the spreader. Assume that the principal axes of the spreader are coincided with the coordinate axes of S-frame, meaning that the moment of inertia matrix $\mathbf{J}$ of the spreader has diagonal form and is denoted by $\mathbf{J}=\operatorname{diag}\left(J_{x}, J_{y}, J_{z}\right)$ in the $\mathrm{S}$-frame. The length and width between the trolley cabling points are denoted by $2 a_{t}$ and $2 b_{t}$, respectively. The length and width between the spreader cabling points are denoted by $2 a$ and $2 b$, respectively. The spreader mass is denoted by $m$. The distance between the trolley and the spreader is denoted by $l$. The elastic modulus and the cross-sectional area of the cable are denoted by $E$ and $A_{c}$, respectively. Let $A_{i}(i=1, \ldots, 4)$ and $B_{i}(i=1, \ldots, 4)$ denote the locations of cables on the trolley and on the spreader, respectively.

The proposed damper system consists of 4 short cables and 8 identical viscous dampers connecting the main cables and the spreader. Each main cable connects with one damper in $X$-direction and another damper in $Y$-direction. Figure 2 shows a typical set of one short cable and two dampers connecting to the main cable number 4 . Denote $D_{i}(i=$ $1, \ldots, 4)$ as the connection points between the main cables and the damper, while $B_{i x}(i=1, \ldots, 4)$ and $B_{i y}(i=1, \ldots, 4)$; denote the connection points between the spreader and the dampers. Two ends of each damper use spherical joints to allow damper to rotate freely. The damping coefficient of each damper is denoted by $c$. The system in Figures 1 and 2 has 18 degrees of freedom, including three spreader translations, three spreader rotations, and three translations of each in four connection points $D_{i}(i=1, \ldots, 4)$. Let $x, y, z$ denote three spreader translations measured from static position, $\phi, \theta, \psi$ denote the angles representing the roll, pitch, and yaw (skew) motions of the spreader, and $x_{i}, y_{i}, z_{i}(i=1, \ldots, 4)$ denote three translations of the connection points $D_{i}(i=1, \ldots, 4)$ measured from static position. The derivation of equations of motion is explained in the appendix. The nonlinear equations are very complicated to write in the full forms. However, the linearized equations can be written in compact forms by using the following fundamental assumption: four main cables are parallel in static position. This assumption is valid if the main crane cables are long enough. Let us introduce the following parameters:

$$
\begin{aligned}
\omega_{s} & =\sqrt{\frac{g}{l}} ; \\
\tau & =\omega_{s} t ; \\
\alpha_{s} & =\sqrt{\frac{E A_{c}}{m g}} ; \\
\zeta & =\frac{c}{2 m \omega_{s}} ;
\end{aligned}
$$




$$
\begin{aligned}
& \gamma=\frac{l-l_{d}}{l} ; \\
& d=\frac{u_{d}}{l} ; \\
& \beta=\frac{u_{d}^{2}}{u_{d}^{2}+l_{d}^{2}} ; \\
& r_{a}=\frac{a}{l} ; \\
& r_{b}=\frac{b}{l} \\
& J_{1}=\frac{J_{x}}{m l^{2}} ; \\
& J_{2}=\frac{J_{y}}{m l^{2}} ; \\
& J_{3}=\frac{J_{z}}{m l^{2}}
\end{aligned}
$$

in which, $\omega_{s}$ is the spreader sway frequency, $\tau$ is the normalized time with time scale $\omega_{s}^{-1}$ and the dot operator from now denoting the differentiation with respect to $\tau, \alpha_{s}$ is the ratio between the vertical vibration frequency and the sway frequency, $\zeta$ is the damping ratio of each viscous damper, $\gamma$ is the location parameter, $d, r_{a}, r_{b}$ are normalized lengths, $J_{i}(i=1, \ldots, 3)$ are normalized moment inertias, and $\beta$ is the cosine of the angle between damper and spreader. The linearized equations can be expressed in vector matrix form as follows:

$$
\begin{aligned}
{\left[\begin{array}{cccc}
\mathbf{M}_{1} & \mathbf{0} & \mathbf{0} & 0 \\
\mathbf{0} & \mathbf{0} & \mathbf{0} & \mathbf{0} \\
\mathbf{0} & \mathbf{0} & \mathbf{M}_{3} & \mathbf{0} \\
\mathbf{0} & \mathbf{0} & \mathbf{0} & \mathbf{0}
\end{array}\right]\left[\begin{array}{l}
\ddot{\mathrm{s}}_{1} \\
\ddot{\mathrm{s}}_{2} \\
\ddot{\mathrm{s}}_{3} \\
\ddot{\mathrm{s}}_{4}
\end{array}\right] } \\
+2 \zeta \beta\left[\begin{array}{llll}
\mathrm{C}_{1} & \mathrm{C}_{12} & \mathrm{C}_{13} & \mathrm{C}_{14} \\
\mathrm{C}_{12}^{T} & \mathrm{C}_{2} & \mathrm{C}_{23} & \mathrm{C}_{24} \\
\mathrm{C}_{13}^{T} & \mathrm{C}_{23}^{T} & \mathrm{C}_{3} & \mathrm{C}_{34} \\
\mathrm{C}_{14}^{T} & \mathrm{C}_{24}^{T} & \mathrm{C}_{34}^{T} & \mathrm{C}_{4}
\end{array}\right]\left[\begin{array}{l}
\dot{\mathrm{s}}_{1} \\
\dot{\mathrm{s}}_{2} \\
\dot{\mathrm{s}}_{3} \\
\dot{\mathrm{s}}_{4}
\end{array}\right]
\end{aligned}
$$

$$
+\left[\begin{array}{cccc}
\mathbf{K}_{1} & \mathbf{K}_{12} & \mathbf{0} & \mathbf{0} \\
\mathbf{K}_{12}^{T} & \mathbf{K}_{2} & \mathbf{0} & \mathbf{0} \\
\mathbf{0} & \mathbf{0} & \mathbf{K}_{3} & \mathbf{K}_{34} \\
\mathbf{0} & \mathbf{0} & \mathbf{K}_{34}^{T} & \mathbf{K}_{4}
\end{array}\right]\left[\begin{array}{l}
\mathbf{s}_{1} \\
\mathbf{s}_{2} \\
\mathbf{s}_{3} \\
\mathbf{s}_{4}
\end{array}\right]=\left[\begin{array}{l}
0 \\
0 \\
0 \\
0
\end{array}\right],
$$

in which the superscript " $T$ " denotes transpose matrix and

$$
\begin{aligned}
& \mathbf{s}_{1}=\left[\begin{array}{lll}
x & y & l \psi
\end{array}\right]^{T}, \\
& \mathbf{s}_{\mathbf{2}}=\left[\begin{array}{llllllll}
x_{1} & y_{1} & x_{2} & y_{2} & x_{3} & y_{3} & x_{4} & y_{4}
\end{array}\right]^{T} \text {, } \\
& \mathbf{s}_{\mathbf{3}}=\left[\begin{array}{lll}
z & l \phi & l \theta
\end{array}\right]^{T}, \\
& \mathbf{s}_{4}=\left[\begin{array}{llll}
z_{1} & z_{2} & z_{3} & z_{4}
\end{array}\right]^{T}, \\
& \mathbf{M}_{1}=\operatorname{diag}\left(1,1, J_{3}\right) \text {, } \\
& \mathbf{M}_{3}=\operatorname{diag}\left(1, J_{1}, J_{2}\right) \text {, } \\
& \mathbf{K}_{\mathbf{1}}=\frac{1}{\gamma} \operatorname{diag}\left(1,1, r_{a}^{2}+r_{b}^{2}\right), \\
& \mathbf{K}_{12}=\frac{1}{4 \gamma}\left[\begin{array}{cccccccc}
-1 & 0 & -1 & 0 & -1 & 0 & -1 & 0 \\
0 & -1 & 0 & -1 & 0 & -1 & 0 & -1 \\
r_{b} & -r_{a} & r_{b} & r_{a} & -r_{b} & r_{a} & -r_{b} & -r_{a}
\end{array}\right] \text {, } \\
& \mathbf{K}_{\mathbf{2}}=\frac{1}{4 \gamma(1-\gamma)} \mathbf{I}_{\mathbf{8}} \\
& \mathbf{K}_{\mathbf{3}}=4 \frac{\alpha_{s}^{2}}{\gamma} \operatorname{diag}\left(1, r_{b}^{2}, r_{a}^{2}\right) \text {, } \\
& \mathbf{K}_{\mathbf{3 4}}=\frac{\alpha_{s}^{2}}{\gamma}\left[\begin{array}{cccc}
-1 & -1 & -1 & -1 \\
-r_{b} & -r_{b} & r_{b} & r_{b} \\
r_{a} & -r_{a} & -r_{a} & r_{a}
\end{array}\right] \text {, }
\end{aligned}
$$




$$
\begin{aligned}
& \mathbf{K}_{\mathbf{4}}=\frac{\alpha_{s}^{2}}{\gamma(1-\gamma)} \mathbf{I}_{\mathbf{4}}, \\
& \mathbf{C}_{\mathbf{1}}=4 \times \operatorname{diag}\left(1,1, r_{a}^{2}+r_{b}^{2}\right) \text {, } \\
& \mathbf{C}_{12}=\left[\begin{array}{cccccccc}
-1 & 0 & -1 & 0 & -1 & 0 & -1 & 0 \\
0 & -1 & 0 & -1 & 0 & -1 & 0 & -1 \\
r_{b} & -r_{a} & r_{b} & r_{a} & -r_{b} & r_{a} & -r_{b} & -r_{a}
\end{array}\right] \text {, } \\
& \mathrm{C}_{2}=\mathrm{I}_{8} \text {, } \\
& \mathbf{C}_{13}=\frac{\gamma}{d}\left[\begin{array}{ccc}
0 & 0 & 4\left(r_{a}-d\right) \\
0 & -4\left(r_{b}-d\right) & 0 \\
0 & 0 & 0
\end{array}\right] \\
& \mathbf{C}_{\mathbf{1 4}}=\frac{\gamma}{d}\left[\begin{array}{cccc}
1 & -1 & -1 & 1 \\
1 & 1 & -1 & -1 \\
r_{a}-r_{b} & r_{b}-r_{a} & r_{a}-r_{b} & r_{b}-r_{a}
\end{array}\right] \\
& \mathbf{C}_{23}=\frac{\gamma}{d}\left[\begin{array}{cccccccc}
1 & 1 & -1 & 1 & -1 & -1 & 1 & -1 \\
r_{b} & r_{b}-d & -r_{b} & r_{b}-d & r_{b} & r_{b}-d & -r_{b} & r_{b}-d \\
d-r_{a} & -r_{a} & d-r_{a} & r_{a} & d-r_{a} & -r_{a} & d-r_{a} & r_{a}
\end{array}\right]^{T}, \\
& \mathbf{C}_{24}=\frac{\gamma}{d}\left[\begin{array}{cccccccc}
-1 & -1 & 0 & 0 & 0 & 0 & 0 & 0 \\
0 & 0 & 1 & -1 & 0 & 0 & 0 & 0 \\
0 & 0 & 0 & 0 & 1 & 1 & 0 & 0 \\
0 & 0 & 0 & 0 & 0 & 0 & -1 & 1
\end{array}\right]^{T}, \\
& \mathbf{C}_{3}=4 \frac{\gamma^{2}}{d^{2}} \operatorname{diag}\left(2, r_{b}^{2}+\left(r_{b}-d\right)^{2}, r_{a}^{2}+\left(r_{a}-d\right)^{2}\right), \\
& \mathbf{C}_{34}=\frac{\gamma^{2}}{d^{2}}\left[\begin{array}{cccc}
-2 & -2 & -2 & -2 \\
-2 r_{b}+d & -2 r_{b}+d & 2 r_{b}-d & 2 r_{b}-d \\
2 r_{a}-d & -2 r_{a}+d & -2 r_{a}+d & 2 r_{a}-d
\end{array}\right] \text {, } \\
& \mathbf{C}_{\mathbf{4}}=2 \frac{\gamma^{2}}{d^{2}} \mathbf{I}_{\mathbf{4}}
\end{aligned}
$$

in which $\mathbf{0}$ defines the zeros matrix with appropriate dimension while $\mathbf{I}_{\mathbf{n}}$ denotes the $n \times n$ identity matrix. The motion equations can be simplified more by assumption that the cables are stiff enough to make the heave, the roll, and the pitch motions be small in comparison with the sway and the skew motions. Ignoring the vectors $\mathbf{s}_{\mathbf{3}}$ and $\mathbf{s}_{\mathbf{4}}$ gives the following simplified equations:

$$
\begin{gathered}
{\left[\begin{array}{cc}
\mathbf{M}_{1} & 0 \\
0 & 0
\end{array}\right]\left[\begin{array}{l}
\ddot{\mathbf{s}}_{1} \\
\ddot{\mathbf{s}}_{2}
\end{array}\right]+2 \zeta \beta\left[\begin{array}{ll}
\mathbf{C}_{1} & \mathbf{C}_{12} \\
\mathbf{C}_{12}^{T} & \mathbf{C}_{2}
\end{array}\right]\left[\begin{array}{l}
\dot{\mathbf{s}}_{1} \\
\dot{\mathbf{s}}_{2}
\end{array}\right]} \\
+\left[\begin{array}{cc}
\mathbf{K}_{1} & \mathbf{K}_{12} \\
\mathbf{K}_{12}^{T} & \mathbf{K}_{2}
\end{array}\right]\left[\begin{array}{l}
\mathbf{s}_{1} \\
\mathbf{s}_{2}
\end{array}\right]=\left[\begin{array}{l}
\mathbf{0} \\
\mathbf{0}
\end{array}\right]
\end{gathered}
$$

which can be expressed in state space form as follows:

$$
\dot{\mathbf{p}}=\mathbf{A p},
$$

in which $\mathbf{p}$ is a $14 \times 1$ state vector and $\mathbf{A}$ is a $14 \times 14$ system matrix defined as

$$
\begin{aligned}
& \mathbf{p}=\left[\begin{array}{l}
\mathbf{s}_{1} \\
\dot{\mathbf{s}}_{1} \\
\mathbf{s}_{2}
\end{array}\right] ; \\
& \mathbf{A}=\left[\begin{array}{ccc}
\mathbf{I}_{3} & \mathbf{0} & \mathbf{0} \\
\mathbf{0} & \mathbf{M}_{1} & 2 \zeta \beta \mathbf{C}_{12} \\
\mathbf{0} & \mathbf{0} & 2 \zeta \beta \mathbf{C}_{2}
\end{array}\right]^{-1}\left[\begin{array}{ccc}
\mathbf{0} & \mathbf{I}_{3} & \mathbf{0} \\
\mathbf{K}_{1} & 2 \zeta \beta \mathbf{C}_{1} & \mathbf{K}_{12} \\
\mathbf{K}_{12}^{T} & 2 \zeta \beta \mathbf{C}_{12}^{T} & \mathbf{K}_{2}
\end{array}\right] .
\end{aligned}
$$


The state space form (5) is used in damper design, but the original nonlinear full equation is used in numerical simulation to check the accuracies of the simplified equation (5).

\section{Optimal Damping}

Imagine what would happen with the increased damping in the dampers. Without damping, the response obviously is the same as that in the uncontrolled case. Conversely, when the damping becomes infinite, the two connection points of a damper are virtually fused to each other and the result is a crane system with the cable length shorter but without damping again. Therefore, somewhere between these extremes, there must be an optimal damping value. In this section, the optimal value is obtained by minimizing the time integration of the stored energy in sway and skew motions.

In linear region, the stored energy in sway and skew motions, which consists of kinetic and potential energies can be expressed by

$$
E_{s}=\dot{\mathbf{s}}_{\mathbf{1}}^{T} \mathbf{M}_{\mathbf{1}} \dot{\mathbf{s}}_{\mathbf{1}}+\left[\begin{array}{ll}
\mathbf{s}_{\mathbf{1}}^{T} & \mathbf{s}_{2}^{T}
\end{array}\right]\left[\begin{array}{cc}
\mathbf{K}_{\mathbf{1}} & \mathbf{K}_{\mathbf{1 2}} \\
\mathbf{K}_{\mathbf{1 2}}^{T} & \mathbf{K}_{\mathbf{2}}
\end{array}\right]\left[\begin{array}{l}
\mathbf{s}_{\mathbf{1}} \\
\mathbf{s}_{\mathbf{2}}
\end{array}\right]=\mathbf{p}^{T} \mathbf{Q} \mathbf{p},
$$

in which,

$$
\mathbf{Q}=\left[\begin{array}{ccc}
\mathbf{K}_{1} & \mathbf{0} & \mathbf{K}_{12} \\
\mathbf{0} & \mathbf{M}_{\mathbf{1}} & \mathbf{0} \\
\mathbf{K}_{12}^{T} & \mathbf{0} & \mathbf{K}_{2}
\end{array}\right] .
$$

Because the stored energy is reduced over time due to the damping, the integral of energy should be considered. The following nondimensional performance index is introduced:

$$
J\left(\mathbf{p}_{0}, \zeta\right)=\frac{\int_{0}^{\infty} E_{s} d \tau}{E_{s}(0)}=\frac{\int_{0}^{\infty} \mathbf{p}^{T} \mathbf{Q p} d \tau}{\mathbf{p}_{0}^{T} \mathbf{Q} \mathbf{p}_{\mathbf{0}}}
$$

where $\mathbf{p}_{\mathbf{0}}=\mathbf{p}(0)$ is the vector containing initial conditions. The performance index $J$ depends on both initial conditions $\mathbf{p}_{0}$ and damping coefficient $\zeta$. Let us do some transformation to remove the integral. Consider matrix $\mathbf{P}$ being the solution of Lyapunov matrix equation as

$$
\mathbf{P A}+\mathbf{A}^{T} \mathbf{P}+\mathbf{Q}=\mathbf{0} .
$$

The matrix $\mathbf{P}$ can be obtained analytically with the aid of Symbolic Math Toolbox in Matlab. Substituting (10) into the integral in (9) and using state space equation (5) give

$$
\begin{aligned}
\int_{0}^{\infty} \mathbf{p}^{T} \mathbf{Q p} d \tau & =-\int_{0}^{\infty} \mathbf{p}^{T}\left(\mathbf{P A}+\mathbf{A}^{T} \mathbf{P}\right) \mathbf{p} d \tau \\
& =-\int_{0}^{\infty}\left(\mathbf{p}^{T} \mathbf{P} \dot{\mathbf{p}}+\dot{\mathbf{p}}^{T} \mathbf{P} \mathbf{p}\right) d \tau \\
& =\left.\left(\mathbf{p}^{T} \mathbf{P} \mathbf{p}\right)\right|_{\infty} ^{0} \\
& =\mathbf{p}_{\mathbf{0}}^{T} \mathbf{P} \mathbf{p}_{\mathbf{0}}^{T}-\mathbf{p}(\infty)^{T} \mathbf{P} \mathbf{p}(\infty)
\end{aligned}
$$

It is assumed that the system is asymptotically stable due to the presence of damping, we have $\mathbf{p}(\infty)=0$ and the performance index (9) can be written without integral as follows:

$$
J\left(\mathbf{p}_{\mathbf{0}}, \zeta\right)=\frac{\mathbf{p}_{\mathbf{0}}^{T} \mathbf{P} \mathbf{p}_{\mathbf{0}}}{\mathbf{p}_{0}^{T} \mathbf{Q} \mathbf{p}_{\mathbf{0}}} .
$$

Let us find the extrema of $J$ over the change of initial condition $\mathbf{p}_{\mathbf{0}}$. Equation (12) can be rewritten as

$$
\mathbf{p}_{\mathbf{0}}^{T}\left(\mathbf{P}-J\left(\mathbf{p}_{\mathbf{0}}, \zeta\right) \mathbf{Q}\right) \mathbf{p}_{\mathbf{0}}=0 .
$$

Use vector and matrix derivative [18], taking the derivative of (13) with respect to vector $\mathbf{p}_{\mathbf{0}}$ gives

$$
2\left(\mathbf{P}-J\left(\mathbf{p}_{\mathbf{0}}, \zeta\right) \mathbf{Q}\right) \mathbf{p}_{\mathbf{0}}+\mathbf{Q} \mathbf{p}_{\mathbf{0}} \mathbf{p}_{\mathbf{0}}^{T} \frac{\partial J\left(\mathbf{p}_{\mathbf{0}}, \zeta\right)}{\partial \mathbf{p}_{\mathbf{0}}}=\mathbf{0}
$$

By denoting $J_{m}$ as the extremum value of $J$ over the change of initial condition $\mathbf{p}_{\mathbf{0}}$, at the extremum point, we have

$$
\left.\frac{\partial J\left(\mathbf{p}_{0}, \zeta\right)}{\partial \mathbf{p}_{\mathbf{0}}}\right|_{J=J_{m}}=\mathbf{0} .
$$

Equations (14) and (15) show that $J_{m}$ is exactly an eigenvalue of the general eigenvalue problem as

$$
\operatorname{det}\left(\mathbf{P}-J_{m} \mathbf{Q}\right)=0 .
$$

In brief, since the matrix $\mathbf{P}$ has 14 eigenvalues, there are 14 extrema of $J$, which are denoted by $J_{m i}(i=1, \ldots, 14)$. The damping ratio $\zeta$ is found to minimize the average value of the extrema $J_{m i}(i=1, \ldots, 14)$; that is, the optimization problem is

$$
J_{\mathrm{opt}}=\min _{\zeta}\left(\frac{1}{14} \sum_{i=1}^{14} J_{m i}\right) .
$$

It is well known that the trace of a square matrix is exactly equal to the sum of all its eigenvalues. Therefore we have

$$
\sum_{i=1}^{14} J_{m i}=\operatorname{trace}\left(\mathbf{Q}^{-1} \mathbf{P}\right) .
$$

With the aid of Symbolic Math Toolbox in Matlab, the optimal condition,

$$
\frac{\partial}{\partial \zeta}\left(\sum_{i=1}^{14} J_{m i}\right)=0,
$$

leads to the following analytical optimal solution:

$$
\begin{aligned}
\zeta_{\mathrm{opt}} & =\frac{\sqrt{2+J_{3} /\left(r_{a}^{2}+r_{b}^{2}\right)}}{4 \beta \gamma \sqrt{(\gamma+2)(6-5 \gamma)}}, \\
J_{\mathrm{opt}} & =\left.\left(\frac{1}{14} \sum_{i=1}^{14} J_{m i}\right)\right|_{\zeta=\zeta_{\mathrm{opt}}} \\
& =\frac{\sqrt{2+J_{3} /\left(r_{a}^{2}+r_{b}^{2}\right)} \sqrt{(\gamma+2)(6-5 \gamma)}}{7 \gamma} .
\end{aligned}
$$




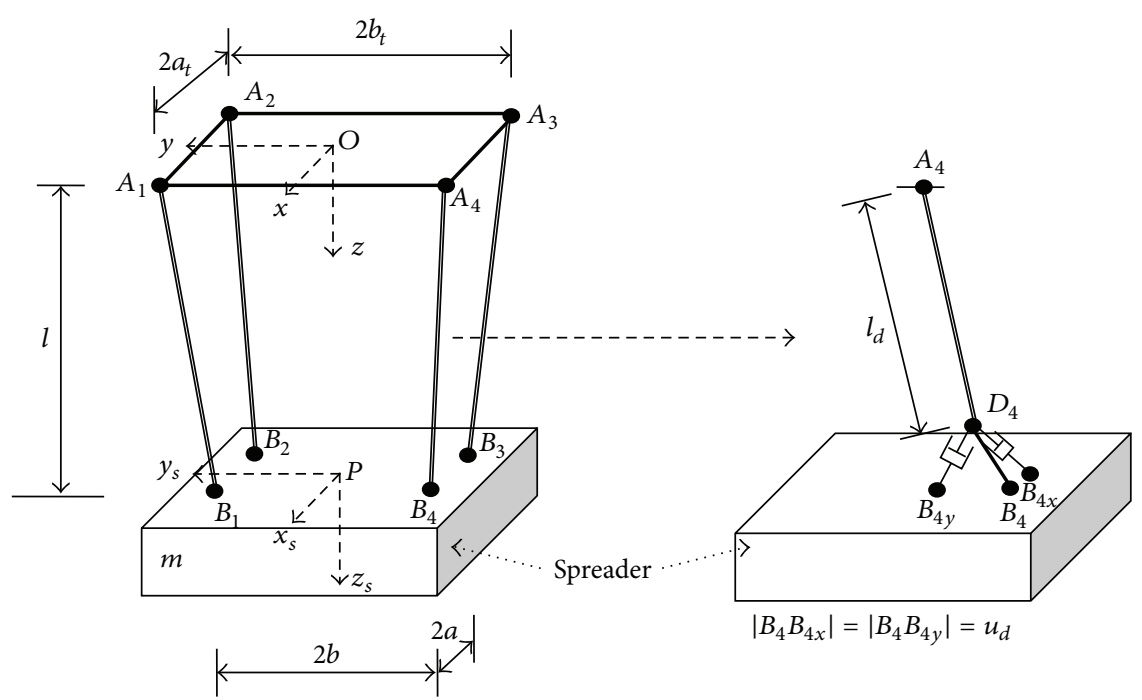

Figure 2: Geometry of the trolley, the spreader, the cables, and a typical cable-damper system connecting to a main cable in work.

The conclusion about the location parameter can be drawn from (21). Actually, the function $\sqrt{(\gamma+2)(6-5 \gamma)} / \gamma$ is monotonic decreasing in the interval from 0 to 1 . Therefore, the optimal performance index decreases with the increase of $\gamma$, which means that the connection point between the damper and the main cable should be as high as possible.

\section{Numerical Demonstration}

4.1. Model Parameters. In order to check the accuracies of simplified linearized equation (5) and to verify the damper effectiveness in original nonlinear system, let us simulate the container crane motion independently by using the software RECURDYN [19]. The model described in the software is completely nonlinear and the design in linear region can be checked. The parameter values of the container crane in Figure 2 are summarized in Table 1.

4.2. Damper Effectiveness in Moving Spreader. The optimal damping ratio (20) is obtained for free linear vibration. In fact, the crane vibration can be a combination of forced vibration due to the trolley motions and free vibration due to the initial conditions. While the free vibration can be reduced by the damper, the forced vibration can be reduced more by other active control schemes. In this example, we try to combine the damper with the input shaping method [9-11] to provide the control technique, which does not rely on sensors. The "zero vibration and derivative" (ZVD) input shaper [10] is used. It is also noted that although the input shaping methods can reduce forced vibration due to the trolley motions, they lack the ability to handle external disturbances or initial conditions like the damper.

Let us consider the spreader oscillations induced by the complex crane motion. The trolley moving in $X$-direction (in Figure 2) and the hoist are operated simultaneously to induce the spreader sway motions. The predefined velocity, the trajectory, and the trolley position are shown in Figures 3(a), 3(b), and 3(c). The shaped trolley velocity is obtained by applying the ZVD input shaper to the unshaped velocity through convolution operator [10]. The ZVD input shaped command has a delay of one vibration period in comparison with the unshaped command (as shown in Figures 3(a) and $3(c))$. However, this time penalty is often a small cost in exchange for reducing vibration.

To demonstrate the damper effectiveness in reducing free vibration in all directions, the initial velocities of the spreader are assumed as follows:

$$
\begin{aligned}
& \dot{x}(0)=\dot{y}(0)=0.1(\mathrm{~m} / \mathrm{s}) ; \\
& \dot{\psi}(0)=0.1(\mathrm{rad} / \mathrm{s}) .
\end{aligned}
$$

The sway and skew motions of the spreader are shown in Figures 4-12 for some cases. The qualitative results are shown in Table 2. Moreover, it is noted that all the active schemes acting to the trolley in the $X$-direction have very little effect on the responses of $Y$-offset or skew angle. In the simulated results given by RECURDYN, this behavior also occurs. The plots of the $Y$-offset or the skew angle in the cases of with or without ZVD input shaper are nearly the same. Therefore, for clarification, we only plot the $Y$-offset and skew angle in the cases of with ZVD input shaper.

\section{Discussion}

From the simulated results, some issues are discussed as follows.

(i) By combining the ZVD input shaper with the proposed damper, both vibrations due to the trolley motions and the initial conditions can be reduced (Figures 4, 7, and 10). As shown in Table 2, the proposed damping system can improve the $X$-offset reduction of the ZVD input shaping method from $69 \%$ to $85 \%$.

(ii) While the active control in $X$-direction cannot reduce the $Y$-offset and skew angle, the proposed system can reduce them well (Figures 5, 6, 8, 9, 11, and 12). 
TABLE 1: Numerical values used in simulation.

\begin{tabular}{lcc}
\hline Parameter & Notation & Value \\
\hline Acceleration of gravity & $g$ & $9.80665\left(\mathrm{~m} / \mathrm{s}^{2}\right)$ \\
\hline Distance between trolley and spreader & $l$ & $\begin{array}{c}\text { Minimum: } 15(\mathrm{~m}) \\
\text { Uaximum: } 25(\mathrm{~m}) \\
\text { Used for design: } 20(\mathrm{~m})\end{array}$ \\
\hline Cable diameter & $d$ & $30(\mathrm{~mm})$ \\
\hline Modulus of elasticity & $E$ & $2 \times 10^{11}\left(\mathrm{~N} / \mathrm{m}^{2}\right)$ \\
\hline Trolley cabling point: length $\times$ width & $2 a_{t} \times 2 b_{t}$ & $5 \times 2.9(\mathrm{~m} \times \mathrm{m})$ \\
\hline Spreader cabling point: length $\times$ width & $2 a \times 2 b$ & $5 \times 2.5(\mathrm{~m} \times \mathrm{m})$ \\
\hline Spreader dimension & $s_{x}, s_{y}, s_{z}$ & $6 \times 2.5 \times 1.25(\mathrm{~m} \times \mathrm{m})$ \\
\hline Spreader mass & $m$ & Used for design: $20($ tons $)$, \\
\hline Spreader inertias in S-frame & $J_{x}, J_{y}, J_{z}$ & taken two values in simulation: $20($ tons), $60($ tons $)$ \\
\hline Length of short cables & $l-l_{d}$ & Taken two values in simulation: $2(\mathrm{~m}), 4(\mathrm{~m})$ \\
\hline $\begin{array}{l}\text { Distance from the short cables to the connection points } \\
\text { between the dampers and the spreader }\end{array}$ & $\left.u_{d}+4 s_{z}{ }^{2}\right) / 12, m\left(s_{x}{ }^{2}+4 s_{z}{ }^{2}\right) / 12, m\left(s_{y}{ }^{2}+s_{x}{ }^{2}\right) / 12$ \\
\hline Optimal damping coefficient & $c_{\mathrm{opt}}$ & $1(\mathrm{~m})$ \\
\hline
\end{tabular}

TABLE 2: Maximum value of the responses after $50 \mathrm{~s}$ (the percentage beside it is the vibration reduction).

\begin{tabular}{lccc}
\hline Case & \multicolumn{2}{c}{ Response } \\
& $X$-offset $(\mathrm{m})$ & $Y$-offset $(\mathrm{m})$ & $0.16(0 \%)$ \\
Without ZVD and damper & $0.52(0 \%)$ & $0.16(0 \%)$ & $0.10(0 \%)$ \\
With ZVD & $0.16(69 \%)$ & $0.06(63 \%)$ & $0.10(0 \%)$ \\
With ZVD and damper $(m=20$ tons, $\gamma=0.1)$ & $0.08(85 \%)$ & $0.09(44 \%)$ & $0.02(80 \%)$ \\
With ZVD and damper $(m=60$ tons, $\gamma=0.1)$ & $0.07(87 \%)$ & $0.01(94 \%)$ & $0.03(70 \%)$ \\
With ZVD and damper $(m=20$ tons, $\gamma=0.2)$ & $0.06(88 \%)$ & & $0.002(98 \%)$ \\
\hline
\end{tabular}

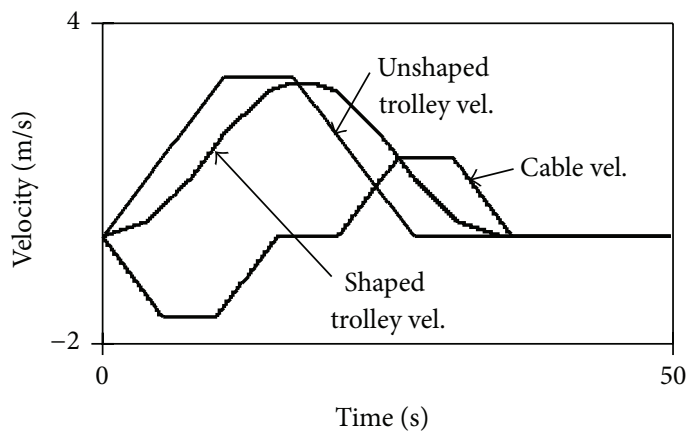

(a)

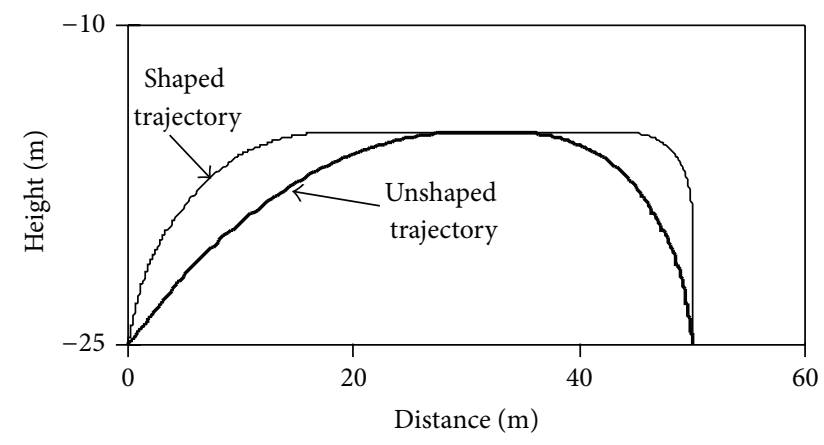

(b)

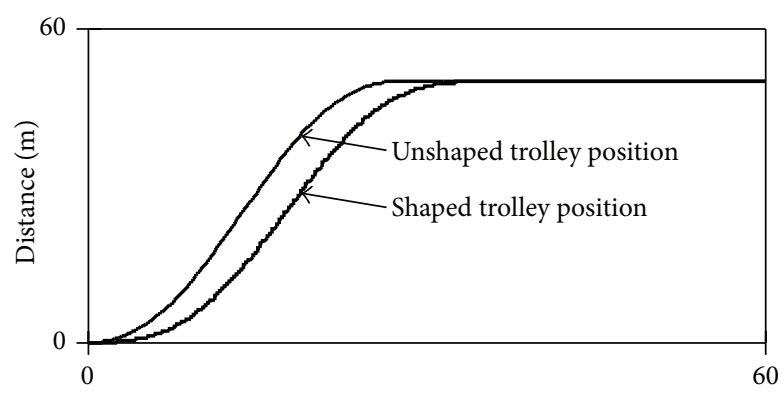

Time $(\mathrm{s})$

(c)

Figure 3: (a) The predefined velocity, (b) the predefined trajectory, (c) and the predefined trolley motion. 


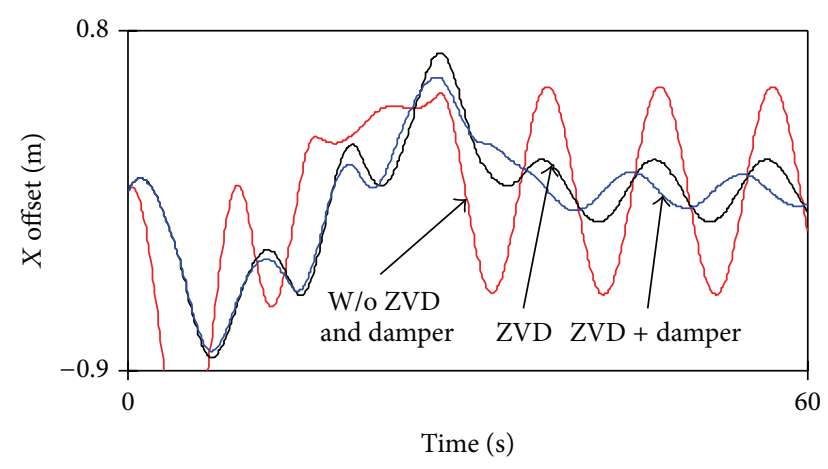

FIgURE 4: Spreader $X$-offset in the case $m=20$ tons, $\gamma=0.1$.

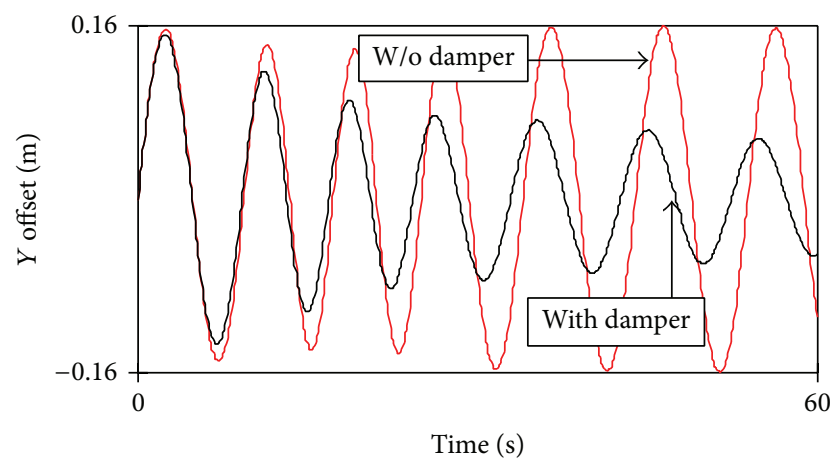

FIGURE 5: Spreader $Y$-offset in the case $m=20$ tons, $\gamma=0.1$.

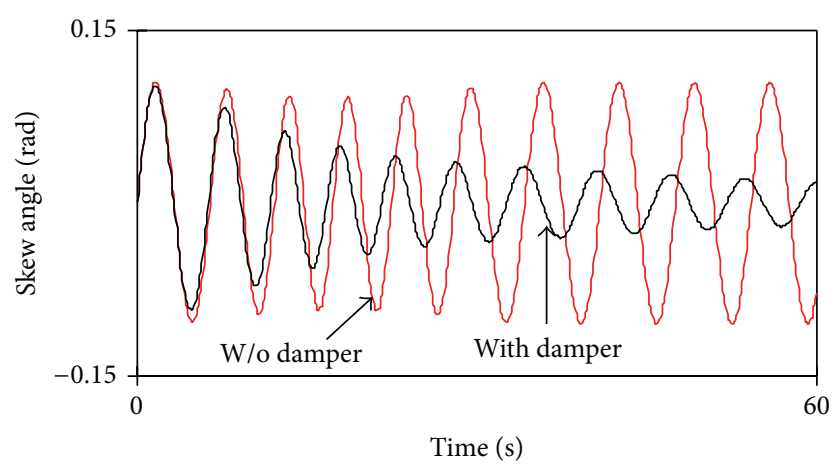

FIGURE 6: Spreader skew angle in the case $m=20$ tons, $\gamma=0.1$.

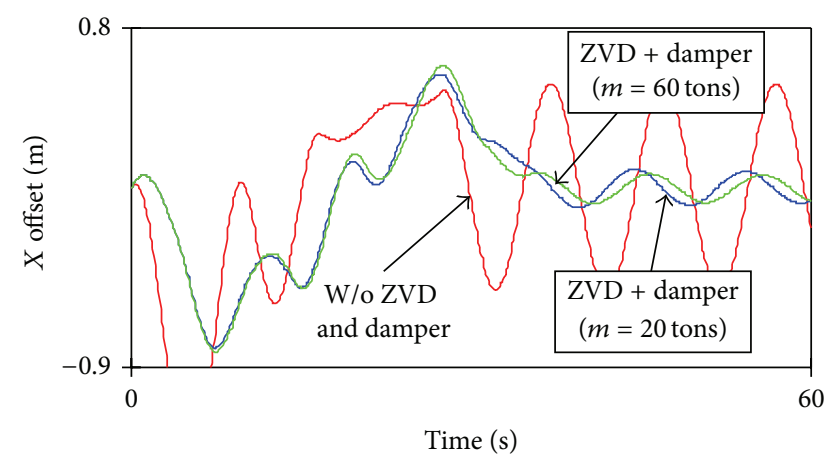

Figure 7: Effect of mass variance on the spreader $X$-offset.

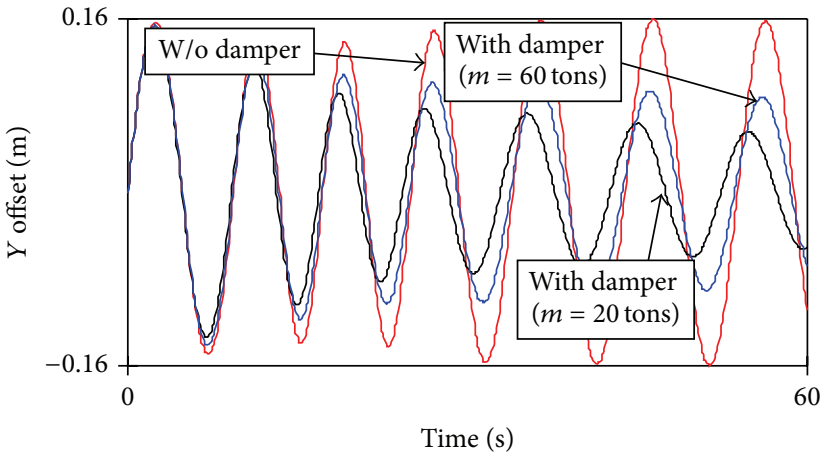

FIGURE 8: Effect of mass variance on the spreader $Y$-offset.

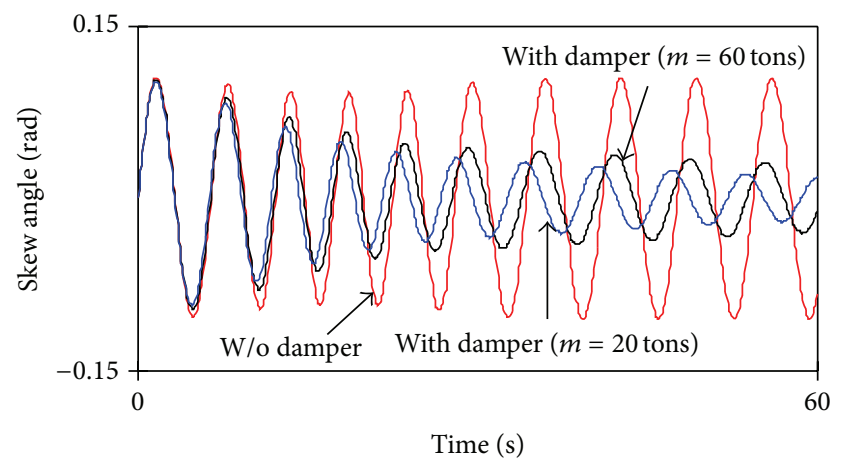

FIGURE 9: Effect of mass variance on the spreader skew angle.

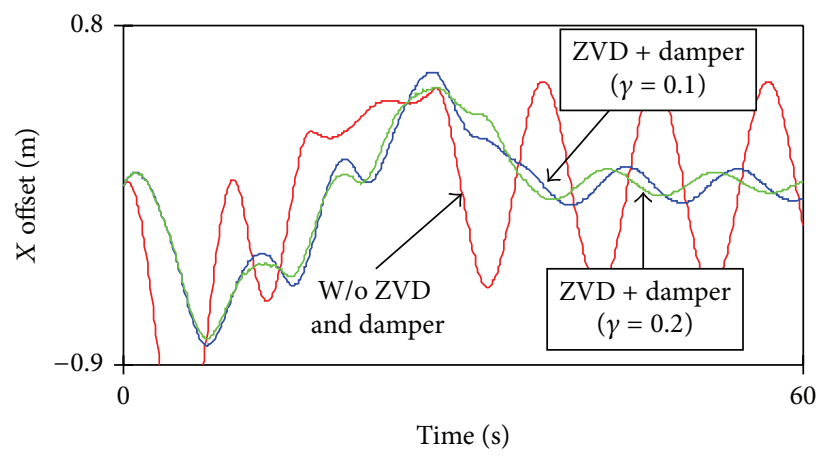

Figure 10: Effect of location parameter on the spreader $X$-offset.

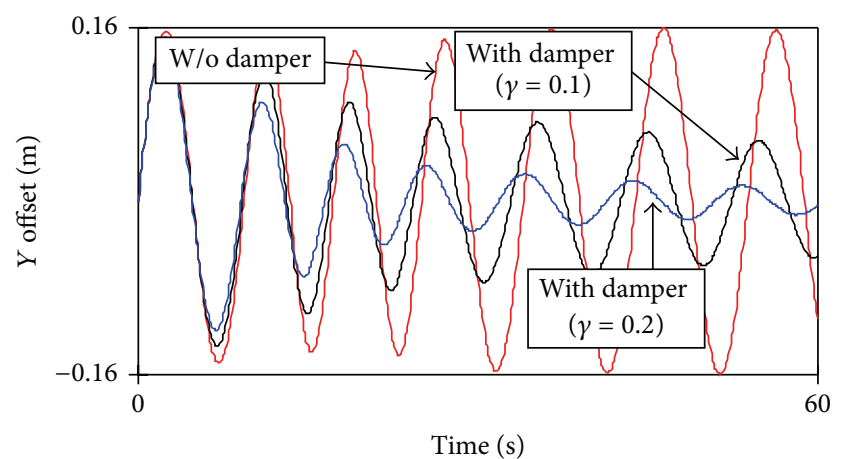

FIGURE 11: Effect of location parameter on the spreader $Y$-offset. 


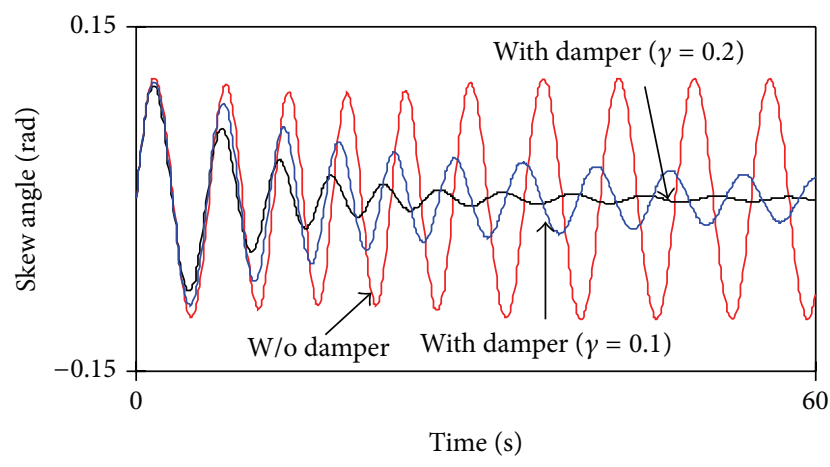

FIGURE 12: Effect of location parameter on the spreader skew angle.

(iii) The damper is designed for the spreader mass of 20 tons. In the case of three-time larger mass $(m=$ 60 tons), the designed damper can have not enough damping and the vibration reduction is poorer than one of the case $m=20$ tons. In the future, the robust design of the damper should be considered.

(iv) The effect of the location parameter $\gamma$ (denoted in (1)) is predicted in the end of Section 3 and is verified in the results in Table 2. The larger value of $\gamma$, the better vibration reduction.

\section{Conclusions}

This paper approaches the antisway and antiskew control of a four-cable suspended crane spreader by introducing a passive damping system. By considering the actual mechanism of a multicable crane, a set of four short cables and eight viscous dampers is introduced to connect the main crane cables and the spreader. The deformation angles between the cables and the spreader in spreader oscillation provide the energy dissipation in the dampers. The optimal damping coefficient of the dampers can be found analytically by minimizing the stored energy of sway and skew motions. Numerical simulations on a crane moving in a predefined trajectory show that the proposed cable-damper system can improve the active control using input shaping technique and is effective in reducing both spreader sway and skew motions.

\section{Appendix}

\section{Derivation of Equations of Motion}

Because there are totally 8 dampers and 8 cable parts, it is not convenient if the forces in each part are written in detail. Instead of that, the forces in the general links as shown in Figure 13 are calculated. Two general links consist of the link $A D$ representing the main cable and the link $D B$ representing the short cable or the viscous damper. Two coordinate reference frames are assigned. They are the global reference frame $(O x y z)$ (called T-frame), on the base of the rail, and the spreader attached reference frame $\left(P x_{s} y_{s} z_{s}\right)$ (called Sframe), in the geometric center of the top of the spreader. In the T-frame, the coordinates of points $A, D, P$ are denoted as

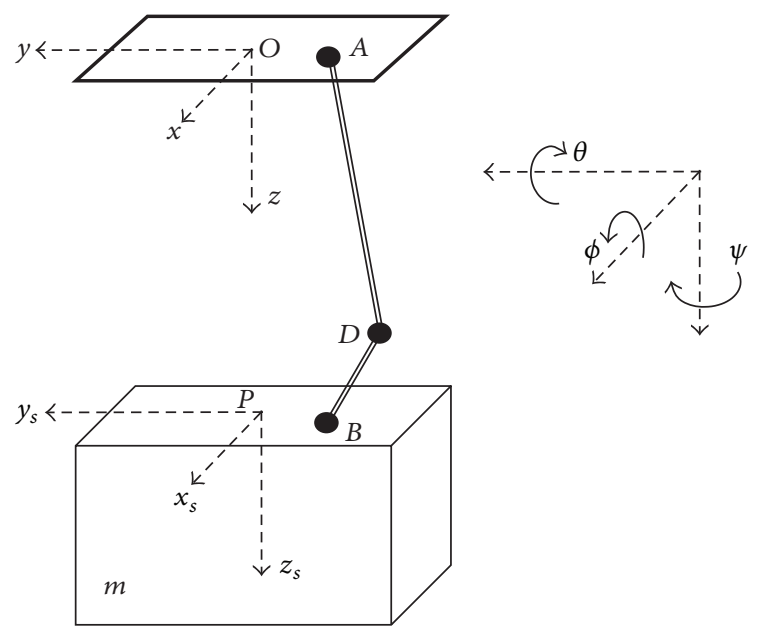

FIgURE 13: Two general links connect the trolley and the spreader and three Euler angles.

$A\left(x_{A}, y_{A}, 0\right), D\left(x_{D}, y_{D}, z_{D}\right), P\left(x_{P}, y_{P}, z_{P}\right)$. In the S-frame, the coordinates of point $B$ are denoted as $B\left(x_{B}, y_{B}, 0\right)$.

The 3-2-1 choice of Euler angles is used to derive the motion equations. Three successive elementary rotations defined by the yaw angle $\psi$, the pitch angle $\theta$, and the roll angle $\phi$ are shown in Figure 13. The coordinate transformation matrix from the S-frame to the T-frame is defined as follows:

$\mathbf{R}$

$$
\begin{aligned}
& =\mathbf{R}(z, \psi) \mathbf{R}(y, \theta) \mathbf{R}(x, \phi) \\
& =\left[\begin{array}{ccc}
\cos \psi & -\sin \psi & 0 \\
\sin \psi & \cos \psi & 0 \\
0 & 0 & 1
\end{array}\right]\left[\begin{array}{ccc}
\cos \theta & 0 & \sin \theta \\
0 & 1 & 0 \\
-\sin \theta & 0 & \cos \theta
\end{array}\right]\left[\begin{array}{ccc}
1 & 0 & 0 \\
0 & \cos \phi & -\sin \phi \\
0 & \sin \phi & \cos \phi
\end{array}\right] .
\end{aligned}
$$

A vector $\mathbf{V}$ will be denoted by $\mathbf{V}^{(T)}$ or $\mathbf{V}^{(S)}$ if it is defined in the global reference frame ( $\mathrm{T}$-frame) or in the spreader reference (S-frame), respectively. A vector $\mathrm{V}$ connecting any two points $A$ and $B$ will be denoted by $\mathbf{V}_{A B}$. Some vectors are calculated as follows:

$$
\begin{aligned}
\mathbf{V}_{O A}^{(T)}= & {\left[\begin{array}{lll}
x_{A} & y_{A} & 0
\end{array}\right]^{T}, } \\
\mathbf{V}_{O P}^{(T)}= & {\left[\begin{array}{lll}
x_{P} & y_{P} & z_{P}
\end{array}\right]^{T}, } \\
\mathbf{V}_{P B}^{(T)}= & \mathbf{R} \cdot \mathbf{V}_{P B}^{(S)}=\mathbf{R}\left[\begin{array}{lll}
x_{B} & y_{B} & 0
\end{array}\right]^{T}, \\
\mathbf{V}_{A D}^{(T)}= & {\left[\begin{array}{lll}
x_{D}-x_{A} & y_{D}-y_{A} & z_{D}
\end{array}\right]^{T}, } \\
\mathbf{V}_{D B}^{(T)}= & \mathbf{V}_{O P}^{(T)}+\mathbf{V}_{P B}^{(T)}-\mathbf{V}_{O A}^{(T)}-\mathbf{V}_{A D}^{(T)} \\
= & {\left[\begin{array}{lll}
x_{P} & y_{P} & z_{P}
\end{array}\right]^{T}+\mathbf{R}\left[\begin{array}{lll}
x_{B} & y_{B} & 0
\end{array}\right]^{T} } \\
& -\left[\begin{array}{lll}
x_{D} & y_{D} & z_{D}
\end{array}\right]^{T} .
\end{aligned}
$$


Let us denote $\mathbf{u}_{D B}$ as the unit vector and $\varepsilon_{D B}$ as the stretch of the link $D B$, we have

$$
\begin{aligned}
& \mathbf{u}_{D B}=\frac{\mathbf{V}_{D B}^{(T)}}{\left|\mathbf{V}_{D B}^{(T)}\right|}, \\
& \varepsilon_{A D}=\left|\mathbf{V}_{D B}^{(T)}\right|-\left|\mathbf{V}_{D B}^{(T)}\right|_{0},
\end{aligned}
$$

in which $\left|\mathbf{V}_{D B}^{(T)}\right|_{0}$ is the length of the link $D B$ in static position. In the T-frame, the tension force vector and the torque vector of the link $D B$ are given by

$$
\begin{aligned}
\mathbf{F}_{D B}^{(T)} & =-\left(c_{D B} \dot{\varepsilon}_{D B}+k_{D B} \varepsilon_{D B}\right) \mathbf{u}_{D B}, \\
\mathbf{M}_{D B}^{(T)} & =\mathbf{V}_{D B}^{(T)} \times \mathbf{F}_{D B}^{(T)},
\end{aligned}
$$

where $\times$ denotes the cross-product operation, $c_{D B}$ and $k_{D B}$ are the damping coefficient and the stiffness coefficient of the link $D B$, respectively. The same procedure can be applied to find the tension force vector and the torque vector of any link.

After calculating all the force and torque, let us calculate the kinetic and potential energies of the spreader mass. The spreader angular velocity vector $\boldsymbol{\omega}$ can be expressed in the inverse kinematics form as [20]

$$
=\left[\begin{array}{lll}
\dot{\phi}-\dot{\psi} \sin \theta & \dot{\theta} \cos \phi+\dot{\psi} \cos \theta \sin \phi & -\dot{\theta} \sin \phi+\dot{\psi} \cos \theta \cos \phi
\end{array}\right]^{T} .
$$

The kinetic energy $T$ is given by

$$
T=\frac{1}{2} m\left(\dot{x}^{2}+\dot{y}^{2}+\dot{z}^{2}\right)+\frac{1}{2} \boldsymbol{\omega}^{T} \operatorname{diag}\left(J_{x}, J_{y}, J_{z}\right) \boldsymbol{\omega},
$$

in which "diag" denotes the diagonal matrix Assume that the roll angle and pitch angle are small enough to approximate the spreader height by $l-z$, the potential energy $U$ is expressed as

$$
U=m g(l-z)
$$

in which $g$ is the acceleration of gravity. The Lagrange motion equations for 6 degrees of freedom of spreader motion have forms:

$$
\begin{aligned}
& \frac{d}{d t}\left(\frac{\partial(T-U)}{\partial \dot{x}}\right)-\frac{\partial(T-U)}{\partial x}=F_{x} ; \\
& \frac{d}{d t}\left(\frac{\partial(T-U)}{\partial \dot{y}}\right)-\frac{\partial(T-U)}{\partial y}=F_{y} ; \\
& \frac{d}{d t}\left(\frac{\partial(T-U)}{\partial \dot{z}}\right)-\frac{\partial(T-U)}{\partial z}=F_{z} ; \\
& \frac{d}{d t}\left(\frac{\partial(T-U)}{\partial \dot{\phi}}\right)-\frac{\partial(T-U)}{\partial \phi}=M_{x} ; \\
& \frac{d}{d t}\left(\frac{\partial(T-U)}{\partial \dot{\theta}}\right)-\frac{\partial(T-U)}{\partial \theta}=M_{y} ; \\
& \frac{d}{d t}\left(\frac{\partial(T-U)}{\partial \dot{\psi}}\right)-\frac{\partial(T-U)}{\partial \psi}=M_{z} ;
\end{aligned}
$$

in which $F_{x}, F_{y}$, and $F_{z}$ are three components of the total force of every link connecting to the spreader, $M_{x}, M_{y}$, and $M_{z}$ are there components of the total torque of every link connecting to the spreader. Besides, by assuming that the cable mass and the damper mass are ignored, there are three equations of force equilibrium in each connection point between the dampers and the cables. For example, three force equilibrium equations for point $D$ in Figure 13 can be expressed as

$$
F_{D x}=F_{D y}=F_{D z}=0
$$

in which $F_{D x}, F_{D y}$, and $F_{D z}$ are three components of the total force of every link connecting to the point $D$. Since there are four connection points between the dampers and the cables, a total of 12 equations can be obtained. Including 6 Lagrange equations (A.8), the mechanical system has 18 equations for 18 degrees of freedom. The linearized equations can be derived by assuming that the vibration states have small perturbations from their values at the equilibrium point. With the aid of Symbolic Math Toolbox in Matlab, after some manipulations to the first order, the linearized equations can be written in matrix form (2).

\section{Conflict of Interests}

The authors declare that there is no conflict of interests regarding the publication of this paper.

\section{Acknowledgments}

The first author was supported by the second stage of the Brain Korea 21 Project and by Vietnam National Foundation for Science and Technology Development (NAFOSTED) under Grant no. "107.01-2013.18."

\section{References}

[1] Q. H. Ngo and K.-S. Hong, "Skew control of a quay container crane," Journal of Mechanical Science and Technology, vol. 23, no. 12, pp. 3332-3339, 2010.

[2] D.-H. Kim and J.-W. Lee, "Model-based PID control of a crane spreader by four auxiliary cables," Proceedings of the IMechE Part C: Journal of Mechanical Engineering Science, vol. 220, no. 8, pp. 1151-1165, 2006.

[3] H. H. Lee, Y. Liang, and D. Segura, "A sliding-mode antiswing trajectory control for overhead cranes with high-speed load hoisting," Transactions of the ASME-Journal of Dynamic Systems, Measurement and Control, vol. 128, no. 4, pp. 842-845, 2006.

[4] K. L. Sorensen, W. Singhose, and S. Dickerson, "A controller enabling precise positioning and sway reduction in bridge and gantry cranes," Control Engineering Practice, vol. 15, no. 7, pp. 825-837, 2007.

[5] J. Neupert, E. Arnold, K. Schneider, and O. Sawodny, "Tracking and anti-sway control for boom cranes," Control Engineering Practice, vol. 18, no. 1, pp. 31-44, 2010.

[6] N. Uchiyama, "Robust control of rotary crane by partial-state feedback with integrator," Mechatronics, vol. 19, no. 8, pp. 12941302, 2009. 
[7] H. Kawai, Y. B. Kim, and Y. W. Choi, "Anti-sway system with image sensor for container cranes," Journal of Mechanical Science and Technology, vol. 23, no. 10, pp. 2757-2765, 2009.

[8] H. M. Omar and A. H. Nayfeh, "Anti-swing control of gantry and tower cranes using fuzzy and time-delayed feedback with friction compensation," Shock and Vibration, vol. 12, no. 2, pp. 73-89, 2005.

[9] J. Lawrence and W. Singhose, "Command shaping slewing motions for tower cranes," Journal of Vibration and Acoustics, vol. 132, no. 1, Article ID 011002, 2010.

[10] J. Vaughan, A. Yano, and W. Singhose, "Comparison of robust input shapers," Journal of Sound and Vibration, vol. 315, no. 4-5, pp. 797-815, 2008.

[11] D. Blackburn, W. Singhose, J. Kitchen et al., "Command shaping for nonlinear crane dynamics," Journal of Vibration and Control, vol. 16, no. 4, pp. 1-25, 2010.

[12] G. Peláez, J. Doval-Gandoy, N. Caparrini, and J. C. GarcíaPrada, "The time delay filtering method for cancelling vibration on overhead transportation systems modelled as a physical pendulum," Shock and Vibration, vol. 14, no. 1, pp. 53-64, 2007.

[13] H. Matsuhisa, H. Kitaura, M. Isono, H. Utsuno, J. G. Park, and M. Yasuda, "A new Coriolis dynamic absorber for reducing the swing of gondola," in Proceedings of the Asia-Pacific Vibration Conference, pp. 211-215, Langkawi, Malaysia, 2005.

[14] L. D. Viet, N. D. Anh, and H. Matsuhisa, "The effective damping approach to design a dynamic vibration absorber using Coriolis force," Journal of Sound and Vibration, vol. 330, no. 9, pp. 19041916, 2011.

[15] L. D. Viet and Y. Park, "Vibration control of the axisymmetric spherical pendulum by dynamic vibration absorber moving in radial direction," Journal of Mechanical Science and Technology, vol. 25, no. 7, pp. 1703-1709, 2011.

[16] J. B. Klaassens, G. Honderd, A. El Azzouzi, K. C. Cheok, and O. E. Smid, "3D modeling visualization for studying controls of the jumbo container crane," in Proceedings of the American Control Conference (ACC '99), pp. 1754-1758, San Diego, Calif, USA, June 1999.

[17] J.-W. Lee and D.-H. Kim, "Dynamics of a spreader suspended by four flexible cables," Proceedings of the Institution of Mechanical Engineers, Part C: Journal of Mechanical Engineering Science, vol. 218, no. 10, pp. 1125-1138, 2004.

[18] A. Graham, Kronecker Products and Matrix Calculus with Applications, Ellis Horwood, Chichester, UK, 1981.

[19] Function Bay, RecurDyn, Function Bay, 2014, http://www.functionbay.co.kr/.

[20] D. T. Greenwood, Advanced Dynamics, Cambridge University Press, New York, NY, USA, 2003. 

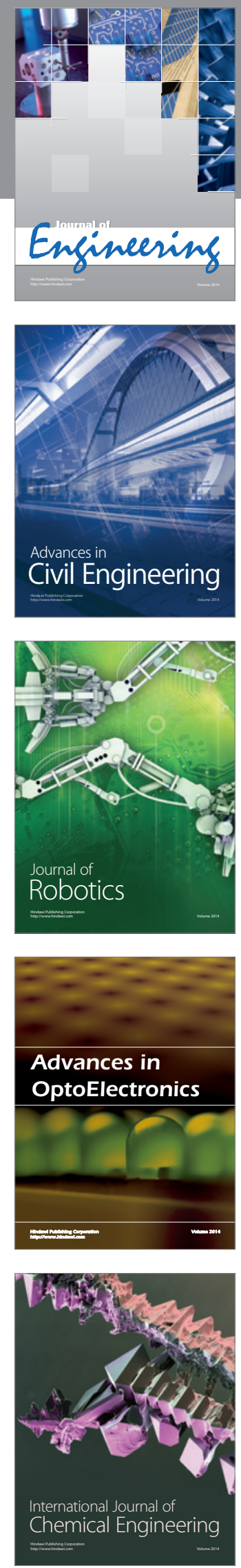

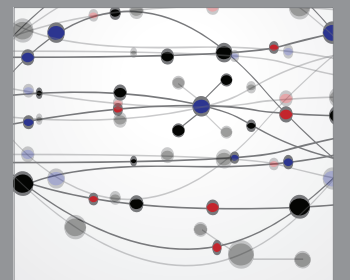

The Scientific World Journal
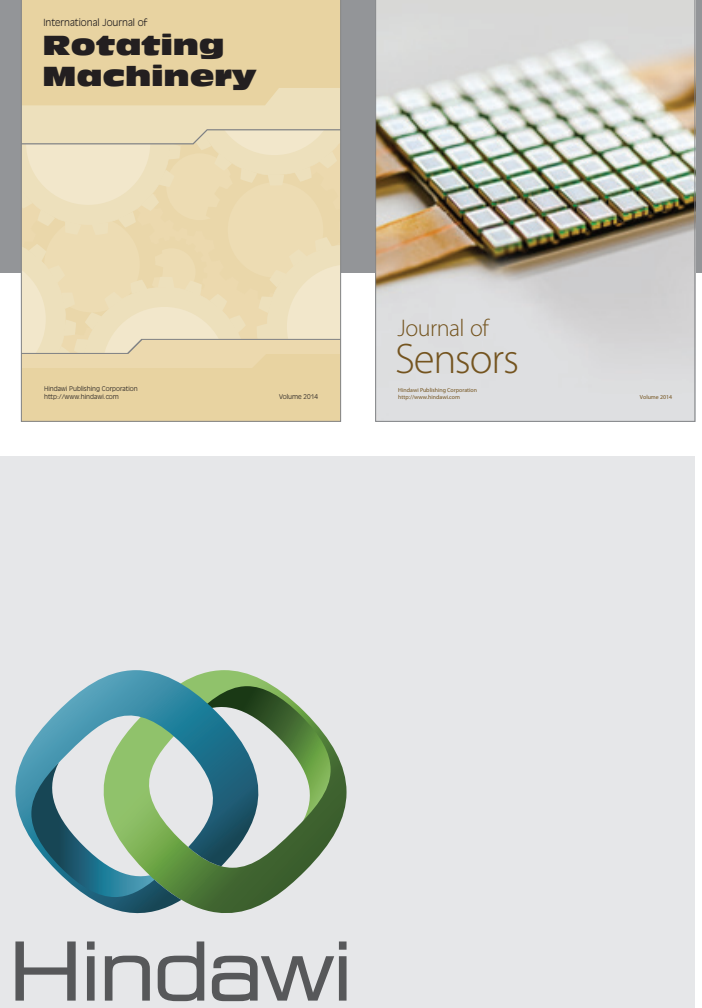

Submit your manuscripts at http://www.hindawi.com
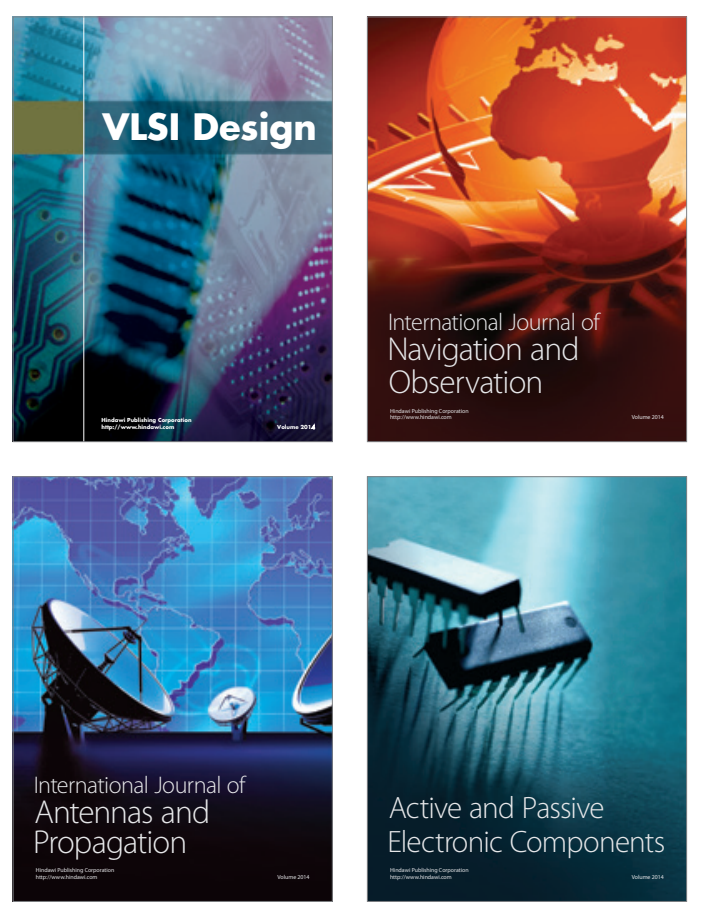
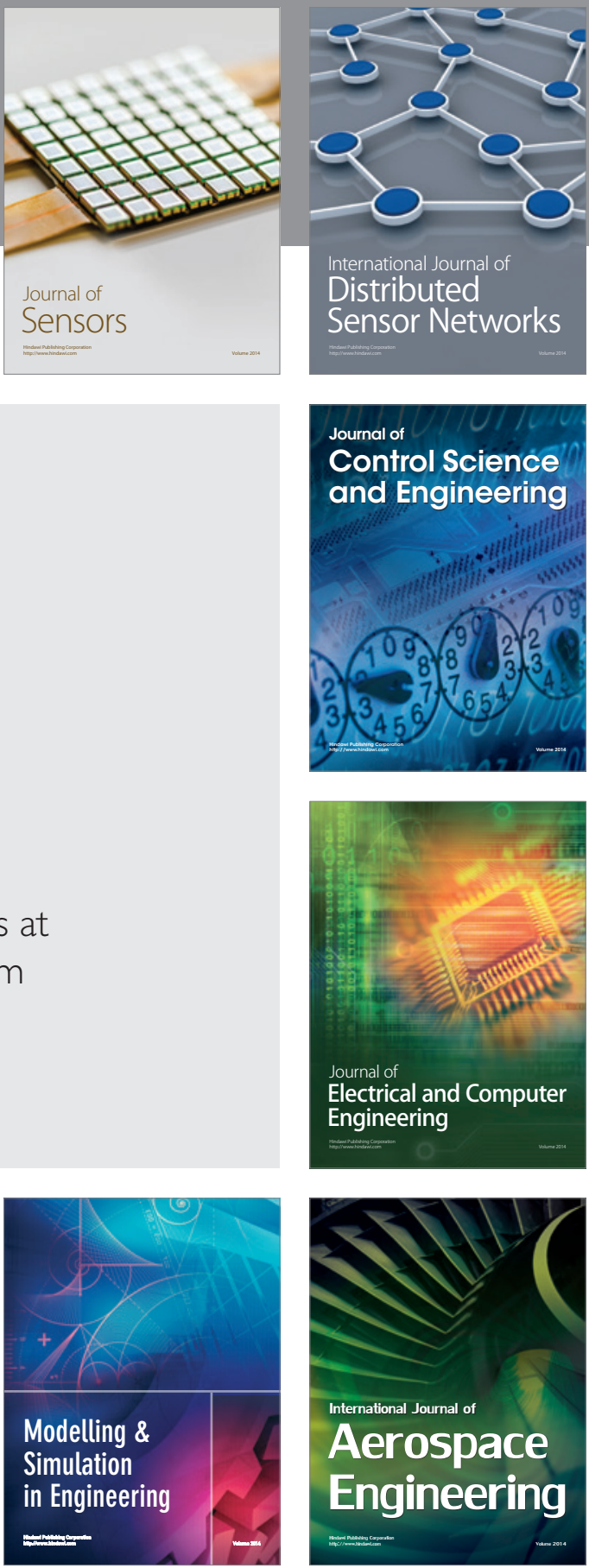

Journal of

Control Science

and Engineering
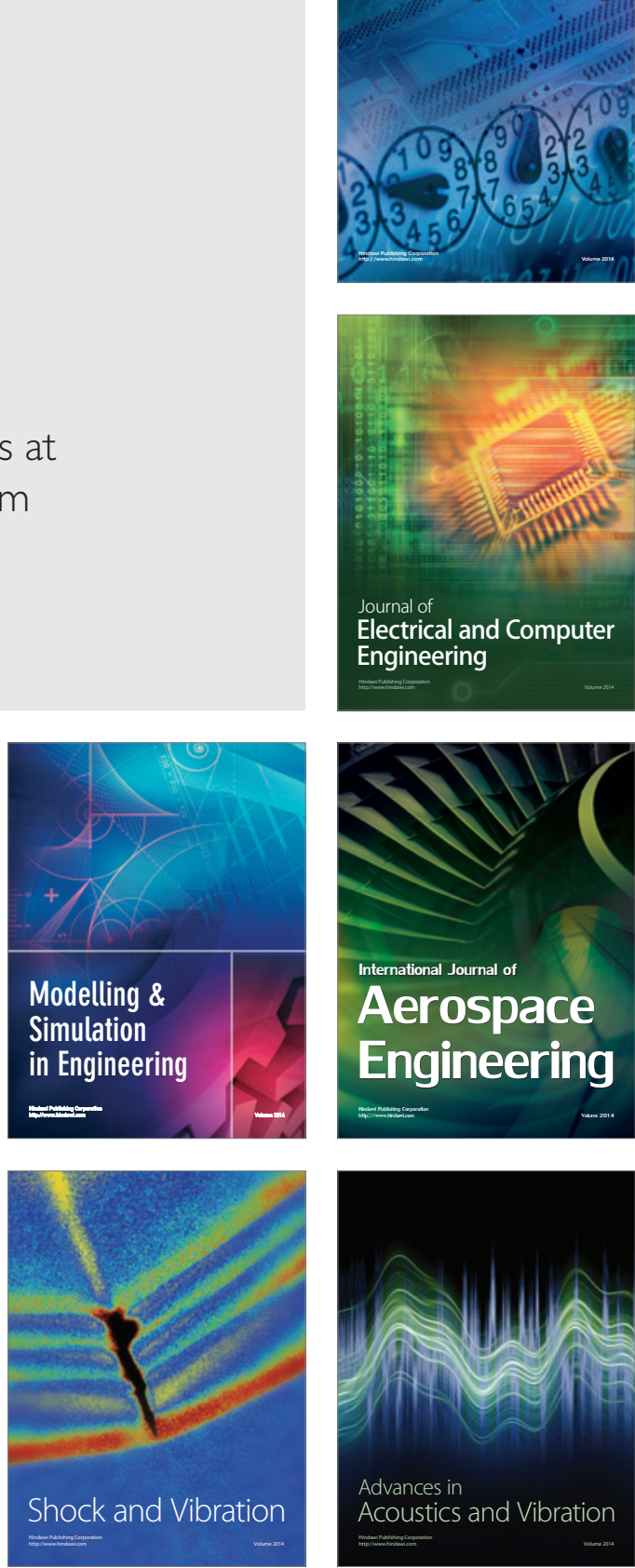